\title{
A new way to hijack the cell
}

DOI:

$10.1038 / \mathrm{nrm} 2318$
The best understood route of endocytosis - the cellular process whereby cells internalize various molecules - is mediated by clathrin, a vesicular coat protein that is used as a membrane deformation scaffold. Recently, however, several clathrin-independent (CI) endocytic pathways have been described, and researchers have been looking (without success) for a coat molecule that would substitute for clathrin function.

In Nature, Johannes and co-workers report an alternative mechanism of CI endocytosis, and show that the B-subunit of the bacterial Shiga toxin $(\mathrm{STxB})$ - which binds
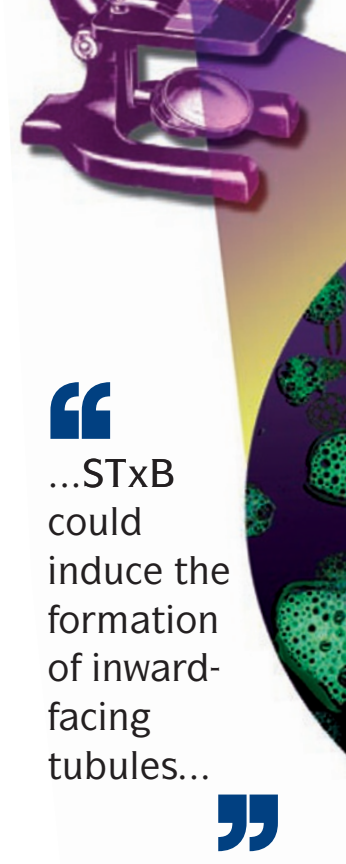

$\rightarrow$ the glycosphingolipid receptor

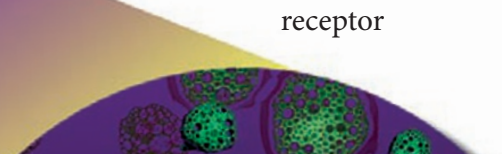

globotriaosyl ceramide (Gb3) - can induce the formation of narrow tubular invaginations on the plasma membrane of target cells without the need for cytosolic factors.

Using various microscopy approaches, the authors monitored changes in cell membrane dynamics during the formation of membrane invaginations, which constitutes the first step of toxin uptake into cells. They observed numerous inward-facing STxB-containing tubular structures of variable length forming at the plasma membranes of cultured cells. The length and number of the tubules increased on energy depletion or on inhibition of dynamin and actin, which are important for the scission of tubules from the cell membrane in other endocytic pathways. These findings suggested that $\mathrm{STxB}$ could induce the formation of inward-

facing tubules, whereas their subsequent scission from the cell membrane required the activity of cellular factors.

To test this hypothesis, Johannes and colleagues reconstituted the process of tubule invagination in a minimal system, using model membranes in the absence of cellular proteins. They showed that tubular structures could only form when STxB was added to the membranes, and clusters of the toxin bound to its receptor (known as STxB-Gb3 cluster domains) were present at sites of membrane invagination before the tubules were detectable.

Is clustering of the toxin a requirement for membrane deformation or a consequence of it? The authors addressed this question by keeping model membranes under high tension, thus preventing tubule formation. They observed that STxB-Gb3 cluster domains could still occur, which demonstrated that this event was not dependent on membrane deformation. Rather, the toxin itself induced the reorganization of membrane lipids before formation of the tubules.

The authors propose that the specific organization of STxB-Gb3 complexes imposes a preferred curvature on the cell membrane that is required for toxin internalization. This cargoinduced mechanism of endocytosis may also apply to invasion by other extracellular pathogens, such as viruses, and to the internalization of other molecules.

Francesca Cesari

ORIGINAL RESEARCH PAPER Römer, W. et al. Shiga toxin induces tubular membrane

invaginations for its uptake into cells. Nature $\mathbf{4 5 0}$ 670-675 (2007)

FURTHER READING Mayor, S. and Pagano, R. E. Pathways of clathrin-independent endocytosis. Nature Rev. Mol. Cell Biol. 8, 603-612 (2007) WEB SITE

Ludger Johannes's laboratory: http://www. curie.fr/recherche/themes/detail equipe.cfm/ lang/ fr/id equipe/101.htm 